\title{
Atom-Probe Tomography of Meteoritic Nanodiamonds.
}

\author{
Philipp R. Heck*1,2 ${ }^{*}$ Dieter Isheim ${ }^{3}$, Michael J. Pellin ${ }^{2,4,5,6}$, Andrew M. Davis ${ }^{1,2,5,6}$, Anirudha V.
}

Sumant $^{7}$, Orlando Auciello ${ }^{8}$, Jeffrey W. Elam ${ }^{9}$, Jon Hiller ${ }^{4}$, David J. Larson ${ }^{10}$, Anil Mane ${ }^{9}$, Surya S. Rout $^{1,2}$, Michael R. Savina ${ }^{2,4}$, David N. Seidman ${ }^{3}$, Thomas Stephan ${ }^{1,2,4,5}$.

${ }^{1}$ Robert A. Pritzker Center for Meteoritics and Polar Studies, The Field Museum of Natural History, Chicago, IL, USA. E-mail: prheck@fieldmuseum.org

${ }^{2}$ Chicago Center for Cosmochemistry, The University of Chicago, Chicago, IL, USA.

${ }^{3}$ Northwestern University Center for Atom-Probe Tomography, Department of Materials Science \& Engineering, Northwestern University, Evanston, IL, USA.

${ }^{4}$ Materials Science Division, Argonne National Laboratory, Argonne, IL, USA.

${ }^{5}$ Department of the Geophysical Sciences, The University of Chicago, Chicago, IL, USA.

${ }^{6}$ Enrico Fermi Institute, The University of Chicago, Chicago, IL, USA.

${ }^{7}$ Center for Nanoscale Materials, Argonne National Laboratory, Argonne, IL, USA.

${ }^{8}$ Department of Materials Science and Engineering and Department of Bioengineering, University of

Texas-Dallas, Richardson, TX, USA.

${ }^{9}$ Energy Systems Division, Argonne National Laboratory, Argonne, IL, USA.

${ }^{10}$ CAMECA Instruments, Inc., Madison, WI, USA.

(*E-mail: prheck@fieldmuseum.org)

Recent advances in instrument development of atom-probe tomography (APT $[1,2]$ ) have revived interest in this analytical technique for the study of extraterrestrial samples. The unmatched combination of atomic level spatial resolution and single-atom detection sensitivity makes it uniquely suited to the study of extraterrestrial nanoparticles such as meteoritic nanodiamonds (ND). Meteoritic ND are 2-3 nm-sized particles that are chemically extracted from primitive meteorites such as carbonaceous chondrites. They were discovered in 1987 in an acid residue of a primitive meteorite [3], but their origin remains a mystery to the present day. Based on their highly anomalous bulk xenon isotopic composition, determined with gas mass spectrometry, they have been considered to have formed prior to the Solar System and incorporated nuclides produced in core collapse supernovae. On the other hand, their bulk carbon and nitrogen isotopic compositions are close to normal and are consistent with an origin in the Solar System [4]. Only single grain analyses of the isotopic compositions of meteoritic ND can reveal if there are different populations with different origins. Because of their small size, single grain analyses are not possible with techniques traditionally used to analyze the isotopic compositions of particles, such as secondary ion mass spectrometry.

In the past five years, we have developed sample preparation procedures and optimized analytical conditions for routine APT analyses of ND [5]. This will allow us to obtain the distribution of the ${ }^{12} \mathrm{C} /{ }^{13} \mathrm{C}$ carbon isotopic ratios of individual ND and will show us if there are different populations of ND from different sources. We used synthetic ND (ultrananocrystalline diamonds and detonation ND) as analytical standards. We discovered an instrumental bias that might be linked to detector dead time [6] that prevented us from obtaining accurate carbon isotopic ratios. Quantification of isotope ratios is ongoing through the study of synthetic ND with normal and doped carbon isotopic compositions. Mass spectra resolving the isotopes of carbon are obtained on a routine basis (Figure 1) as well as atom-byatom three-dimensional tomographic reconstructions (Figure 2). Transmission electron microscopy will be used to constrain the geometry of the tomographic reconstructions. We have not yet detected a significant difference between meteoritic ND and synthetic standards, but we have thus far only 
analyzed a small number of samples. Analyzing a larger number of samples will increase the probability of detecting the full range of carbon isotopic compositions of meteoritic ND. We conclude that APT is an extremely powerful technique for the analysis of extraterrestrial nanoparticles.

\section{References:}

[1] DN Seidman and K Stiller, MRS Bull. 34 (2009) p. 717.

[2] T. F. Kelly and D. J. Larson, Annu. Rev. Mater. Res. 42 (2012) 1.

[3] RS Lewis et al, Nature 326 (1987) p. 160.

[4] SS Russell et al, Meteoritics Planet. Sci. 31 (1996) p. 343.

[5] PR Heck et al, Meteoritics Planet. Sci. (2014) in press.

[6] T Stephan et al, J. Vac. Sci. Technol. A 12 (1994) p. 405.

[7] We thank K. Knight for providing the detonation ND. This work is supported by grants from NASA to PRH and AMD and from the Tawani Foundation and W. H. Ganz III to PRH. Atomic layer deposition was performed at Argonne National Laboratory (ANL). Some UNCD film growth was performed at the Center for Nanoscale Materials at ANL, which is supported by the U.S. Department of Energy, Office of Science, Office of Basic Energy Sciences. Some UNCD film growth and some of the FIB work performed at the ANL was supported by the US Department of Energy, Materials Sciences and Engineering Division. This FIB work was accomplished at the Electron Microscopy Center at ANL, a U.S. Department of Energy Office of Science Laboratory operated by UChicago Argonne, LLC. The LEAP tomograph at NUCAPT was purchased and upgraded with funding from NSF-MRI and ONRDURIP. This research made use of the Shared Facilities at the Materials Research Center of Northwestern University (NU), supported by the NSF's MRSEC program. The Initiative for Sustainability and Energy at Northwestern (ISEN) is acknowledged for grants for upgrades at NUCAPT. Some of the FIB work was performed at the NUANCE Center at NU. NUANCE is supported by NSFNSEC, NSF-MRSEC, Keck Foundation, the State of Illinois, and NU.

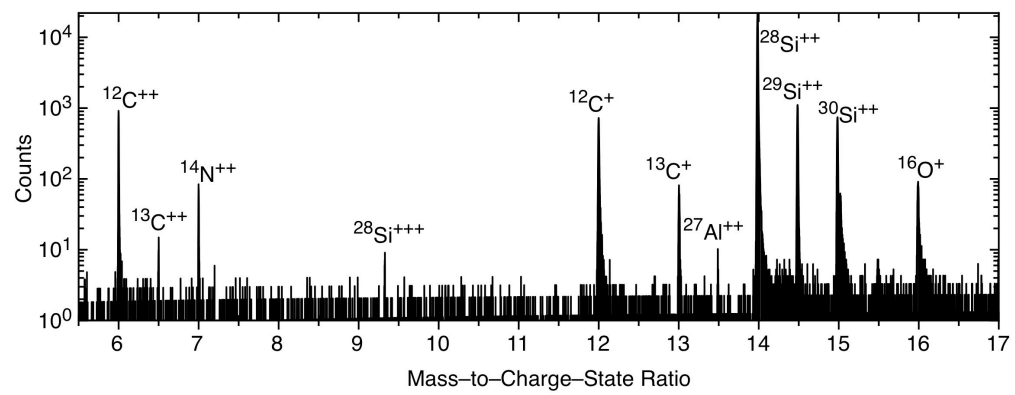

Figure 1. Mass spectrum from APT analyses of synthetic nanodiamonds. Sharp carbon isotope peaks are clearly visible for singly and doubly charged species for ${ }^{12} \mathrm{C}$ and ${ }^{13} \mathrm{C}$. The silicon isotope peaks are from the microtip substrate on which the diamonds reside. Figure from [5].

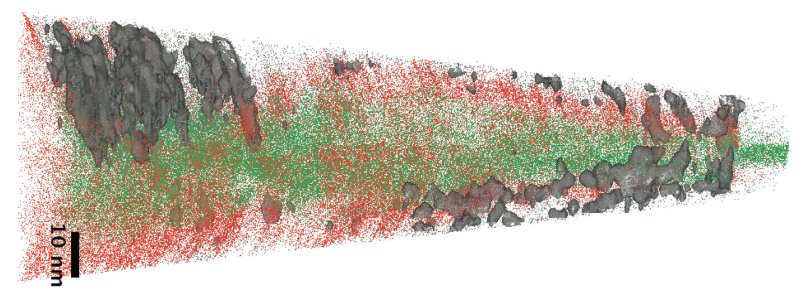

Figure 2. Tomographic 3D reconstruction of a silicon microtip coated with individual and clusters of nanodiamonds from the meteorite Allende (gray shaded areas; Figure from [5]). Each dot represents an atom (carbon gray, oxygen red, silicon green), aspect ratio and scale are approximate. 